\title{
Video Door Phone Surveillance System Using Powerline Communication Channel
}

\author{
Chao-Huang Wei and Shin-An Chen
}

\begin{abstract}
Door phone" is one of the essential communication apparatuses of the modern family. It is used to identify the visitor or for simple voice interlocution. The communication quality of this kind analog phone is not adequate; also, its functionality is also very limited. To cope with the trend of smart home networks development, this work employed a novel powerline communication chip to develop a networked digital video door phone system to replace the conventional ones. They transfer audio visual information and enhance the entrance guarding functions additionally. Three main tasks were developed in this project: construction of an outdoor video phone, construction of an indoor video phone server and porting an embedded operating system for this phone system. The experiment results of this project demonstrate the functionality of this phone system and the user's interaction with smart electrical home appliances over powerline.
\end{abstract}

Index Terms-Android operating system, powerline communication, surveillance system, video door phone.

\section{INTRODUCTION}

Smart Home and Smart Grid are two main issues in today's IT industry. For energy saving and carbon reduction, electrical equipments need not only have theirs own energy-saving design; they should work with surrounding equipments adapting to environmental conditions to achieve better efficiency. Therefore they need a communication channel to hold dialogue with each other and the user in house. The most convenient communication channel in normal household will be the AC power grid [1]-[5].

Door phone is one of the necessary equipment of modern household, but the majority of them are still analog-style phones. These phones have a simple structure and therefore inexpensive, but are used only for voice communication. They also can not be linked into a smart home network system. In this work, a video outdoor phone and its matched indoor video phone server were developed and use power lines as communication media to form a network. Powerline Communication (PLC) is the backbone of a smart home system that enables data, digital voice, image and other control signals transfer over the electrical AC power line. This technology fully utilize the most convenient and popular wiring connection in the house - electric power grid, through many power outlets spread in various rooms via which high-speed data access can be carried out without additional

Manuscript received January 25, 2013; revised March 12, 2013.

C. H. Wei and S. A. Chen are with the Department of Electrical Engineering, Southern Taiwan University of Science and Technology, Tainan, Taiwan (e-mail: drwei@ mail.stust.edu.tw, m9920106@stust.edu.tw). wiring. In addition, the power load monitoring, remote meter reading, smart household appliance control, and etc can be done easily [6]-[8]. Fig. 1 shows possible system architecture of this network.

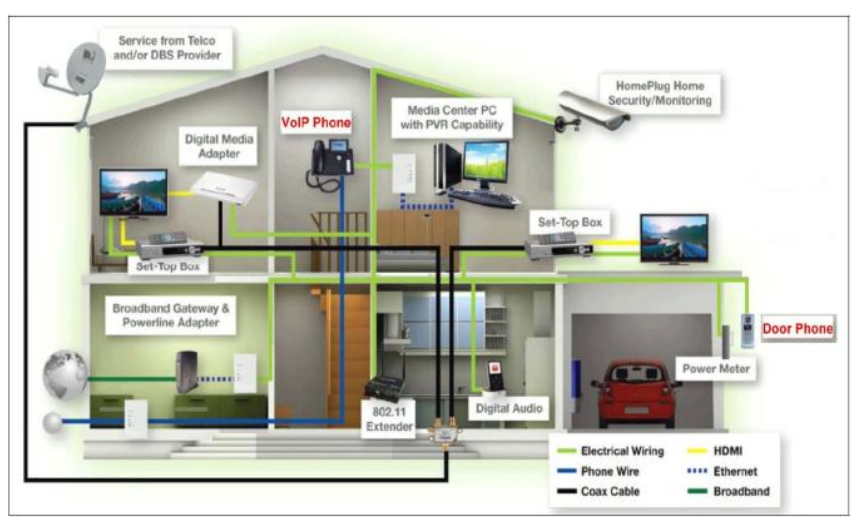

Fig. 1. Architecture of network video phone system [9]

\section{PROCEDURE FOR SySTEM CONSTRUCTION}

\section{A. Construction of Outdoor Video Phone}

Door phone can be used in many places such as single-family building, apartment house, hotels, factories, and communities for identifying the visitors or entrance access control. As mentioned earlier, the video door phone system in this work use power line communication channels to avoid the problems of new network wiring or the unstable wireless signal. By existing in-home power line the indoor telephones, personal computers, and various intelligent appliances can be connected together. The INT6400/INT1400 HomePlug AV chipset from Atheros/Intellon company will be used for network interface (Fig. 2) [9]. This chip is compatible with the latest HomePlug AV standard[10], the mmaximum PHY data rate is up to 200Mbps.

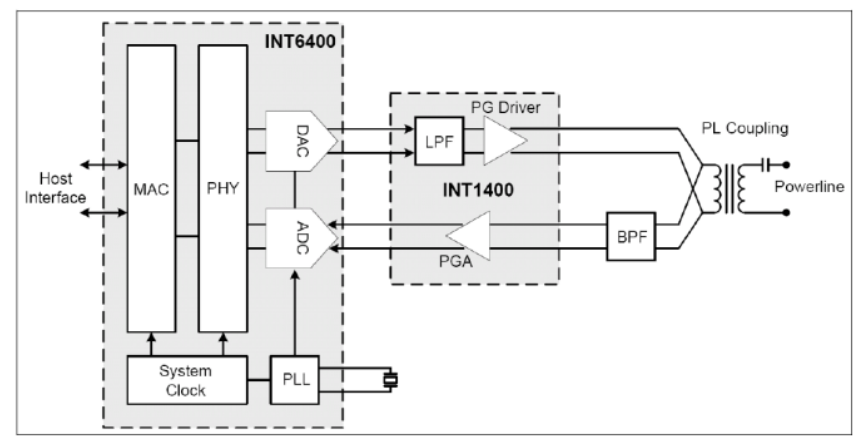

Fig. 2. Internal function block of INT6400/INT1400 HomePlug AV chip[9]

The INT6400 chip contains Medium Access Controller 
(MAC), network Physical Layer, (PHY), ADC, DAC and interfaces for connection to host controller e.g. Media Independent Interface (MII), SPI interface and SDRAM control interface. The INT1400 is an Analogue Frond End (AFE) and line circuit driver chip for power line signal coupling. The digital data output from the physical layer will be converted first by the DAC of INT6400, then through the INT1400 line amplifier driver circuit output to the power cord; on the other side the analog signal on the power line is received through an adjustable gain amplifier of INT1400, then converted through the ADC into a digital data for the network physical layer of IT6400.

The MAC/PHY hardware of IT6400 contains a Data Link Layer (DLL) circuit, a physical layer management circuit, an error correction circuits and a data transceiver. Transmitted data will be fed via encoder to an Interleaver, broken into several packets, then the modulation data packet along with the data frame synchronization signal produced from a Preamble will be sent via an output filter; data will be received via an input filter into demodulation circuit, then the data distributed in separated packets will be re-arranged by a De-Interleaver, after decoding, the data are sent to a management circuit in the physical layer.

The Data Link Layer (DLL) handles following functions related to network transmission:

- Addressing (Network ID and Node ID)

- Send and receive data packet,

- Confirm or deny data transmission,

- Priority setting for pipeline communication access,

- Automatic transmission rate adjustment,

- Adaptive pipeline access of CSMA/CA communication,

- Adaptive backoff algorithm to avoid signal collision,

- Segmentation and reassembly long data packets,

- 16 fragments of data packets (110 bytes 1760 bytes),

- Multi-hop broadcasting,

- Anti-jamming algorithm,

- CNC transfer,

- AES 128-bit encryption.

Media Access Controller (MAC) is used to manage low-level data access, provides several services like pipeline packet transmission logical network addressing, and avoiding signal interference. Especially, the MAC is complied with the HomePlug AV and HomePlug C \& C V1.0 standard[11]-[13]. Due to the maximum effective data length that the PHY can accept is 127 Bytes only, the MAC needs to handle the data splitting process for the long data of upper application layer. The MAC has a unique 16-byte serial number, defines an 11-bit logical node address (Node ID) and a 10-bit network address (Network ID) for network addressing.

Fig. 3 shows the architecture and function blocks of the video door phone, including CMOS camera module, night lighting illumination with infrared lights, speakers and microphone, keypads, operation information and status display with a character type LCD, and an entrance controller with a chip card reader.

The hardware platform will be constructed on a Samsung high-performance 32-bit single-chip S5PC100 [14], this chip is based on ARM CortexA8 high-performance microprocessor. It can operate up to $833 \mathrm{MHz}$, contains $32 \mathrm{~KB}$ data / 32KB instruction cache memory, 256KB L2 cache memory and a rich peripheral interface control circuit (Fig. 4). The S5PC100 processor uses 64-bit internal bus architecture, including many hardware accelerators, such as: dynamic video processing, display control and scaling circuits. The codec supports a variety of formats: MPEF-1/2/4 H263/H264, CV-1, and DivX. The acceleration functions support real-time video conferencing, analog TV output function, and NTSC or PAL mode HDMI. This board provides a 24bit LCD TVout, camera input, audio I/O, serial ports, SD card, SPI, 100M Ethernet network, USB2.0-OTG, USB Host, keypads, and I2C interfaces. It is suitable for high performance embedded systems applications which demand high degree of processing power.

\section{B. Construction of Indoor Video Phone}

Indoor video phone can plug easily in the household power outlet, and link to the video door phone via the power line. Also, the user can touch the graph man-machine interface to send a command to control the door lights, electronic locks, or other smart house appliances. The hardware construction is similar with the video door phone, except that an additional LCD touch screen is required, in order to facilitate the user's operation and display the visitor's images at the door. Application development includes a testing via the power grid to control smart household appliances. The system software structure is shown in Fig. 5, in which the web browser enables the PC connection with the door phone system. The phone software maintenance and updates can be performed via FTP.

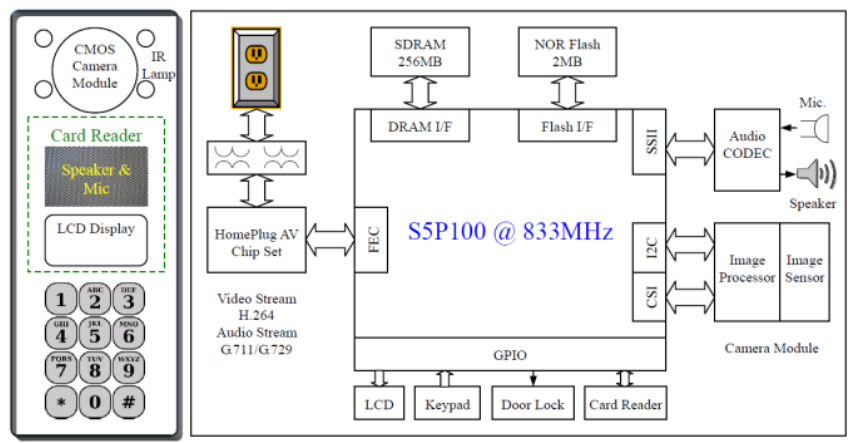

Fig. 3. Hardware architecture of outdoor video phone

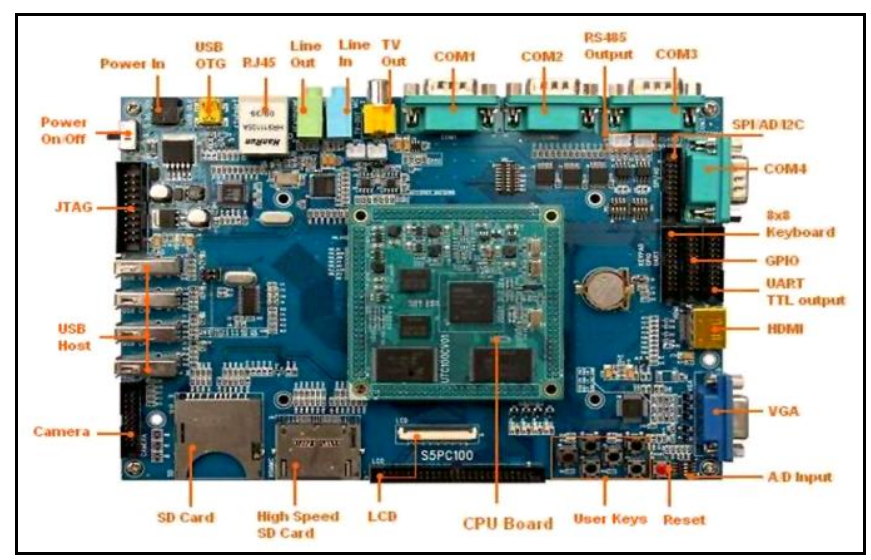

Fig. 4. S5P100 Embedded system development board [14] 


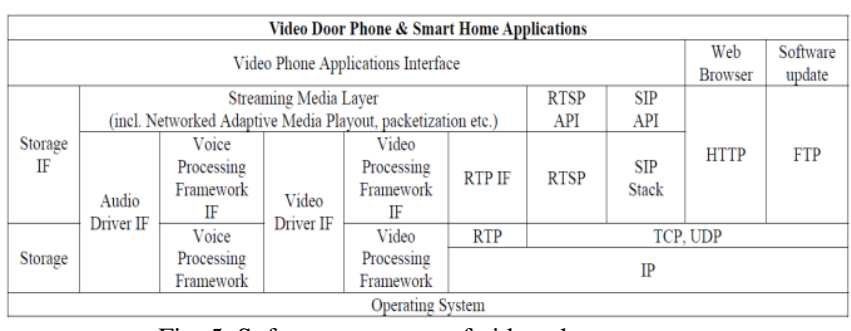

Fig. 5. Software structure of video phone system

The multimedia communications using Session Initiation Protocol (SIP), which is based on the existing Internet multimedia architecture and message transfer capability to provide integrated voice and other multimedia communication services applied to VoIP, video conferencing and instant messaging. The Real Time Streaming Protocol (RTSP) is used to control the audio or video multimedia streaming, allows multiple simultaneous streaming demand control (Multicast) and support for multi-party video conferencing (Video Conference). The Real-time Transport Protocol (RTP) is a protocol in the transport layer to provide one-to-one or one-to-many transmission service, its purpose is to provide timing information for streaming synchronization. The Audio/Video Processing Framework is used to deal with various multimedia codec, playback and streaming. The bottom operating system used is the free and open-source Andriod [15], [16].

\section{Operating System of Video Phone}

Video phone system requires supports by an efficient, real-time operating system, to diversify the function developments and shorten the development cycle. An operating system should provide five major functions:

1) Provide users with easy operating environment,

2) Monitor the entire program processing,

3) Deployment of various hardware resources,

4) File management,

5) Memory management.

The Android multimedia framework contains Java application layer, JAVA framework, C language framework, and hardware abstraction layer [17]. The multimedia content deal with:

- Input and output (audio / video input / output)

- Signal processing (audio / video codec)

Inputs / outputs are realized by the hardware abstraction layer, and the signal is processed by Packet Video. They may use hardware circuits to accelerate performance. The Android multimedia system is shown in Fig. 6, Fig. 7 for Android camera image capture system, and Fig. 8 for Android video player system.

Android multimedia framework uses the Packet Video OpenCORE Platform [26]. It supports all common audio, video, still image formats through the Open Core libraries with the desired multimedia applications, e.g. audio / video capture, playback, video conferencing, instant streaming media players and other applications can be developed quickly.

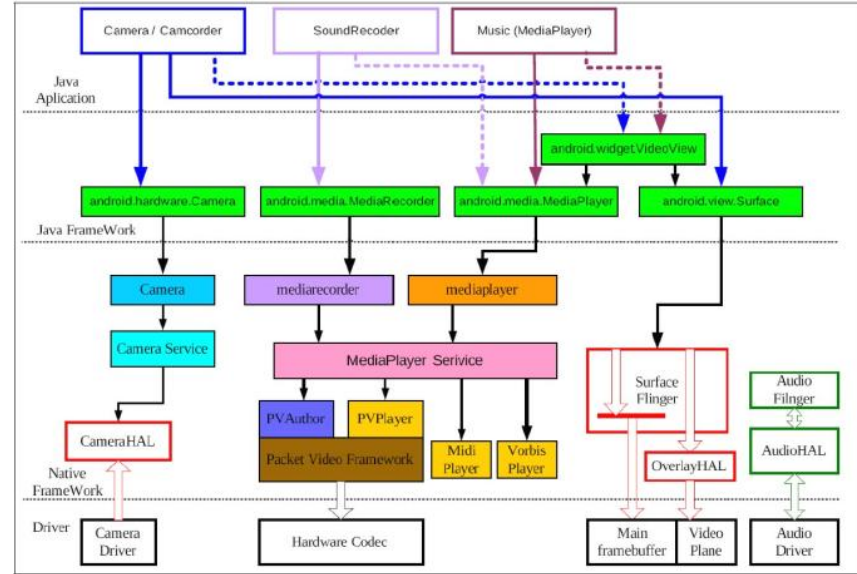

Fig. 6. Android multimedia framework [17]

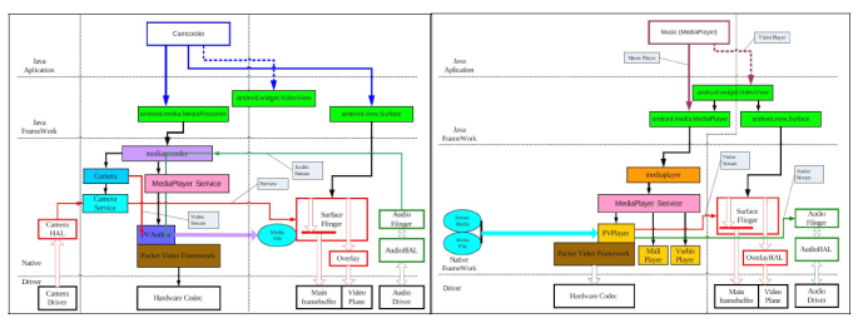

Fig. 7. Camera capture system [17] Fig. 8. AV playback system[17]

The Open Core Multimedia Framework use the OpenMAX 1L interface to extend multimedia codec including playback and download of 3GPP, MPEG-4, AAC and MP3 multimedia file format; download and instant playback of 3GPP, HTTP and RTSP / RTP streaming media; encoding and decoding of MPEG-4, H.263, AVC (H.264), JPEG-motion video and still image; speech coding of AMR-NB and AMR-WB; music encoding of MP3, AAC, and $\mathrm{AAC}+$; video conferencing based on $\mathrm{H} 324-\mathrm{M}$ standard.

\section{TESTING SYSTEM}

Fig. 9 shows the schematic diagram of the testing system in this work. The indoor video phone or computer can link to outdoor video phone via power grid. Users can use the embedded indoor video phone or a remote PC to dialogue with outdoor video phone, and activate the necessary lighting at night or release the electronic door locks. Besides, the user can use the LCD touch panel of indoor video phone to send commands via the electrical network to operate various home appliances connected to this network. The outdoor video phone is equipped with a keypad and a ID reader device that can accept the user's password for control door lock. The images of entrance person will be recorded in the indoor video phone with a time stamp.

Fig. 10 shows the physical structure of the video door phone. The indoor phone is shown at left side, and the outdoor phone is shown at right side. Fig. 11 shows the initial screen of indoor video phone indoor phone after power on; Fig. 12 and Fig. 13 shows the communication between indoor and outdoor phones.

In this work the home appliances monitoring functions via indoor video phone were developed also. The sensing data can be displayed on the user's LCD touch panel, the user can also touch the screen to control home appliances. Commands will be transmitted via the power line to activate or deactivate 
the conventional appliances by the IP network controller. The user control interface of indoor video phone is shown in Fig. 14. Four small lamps in Fig. 15 simulate the power supply to electrical home appliances.

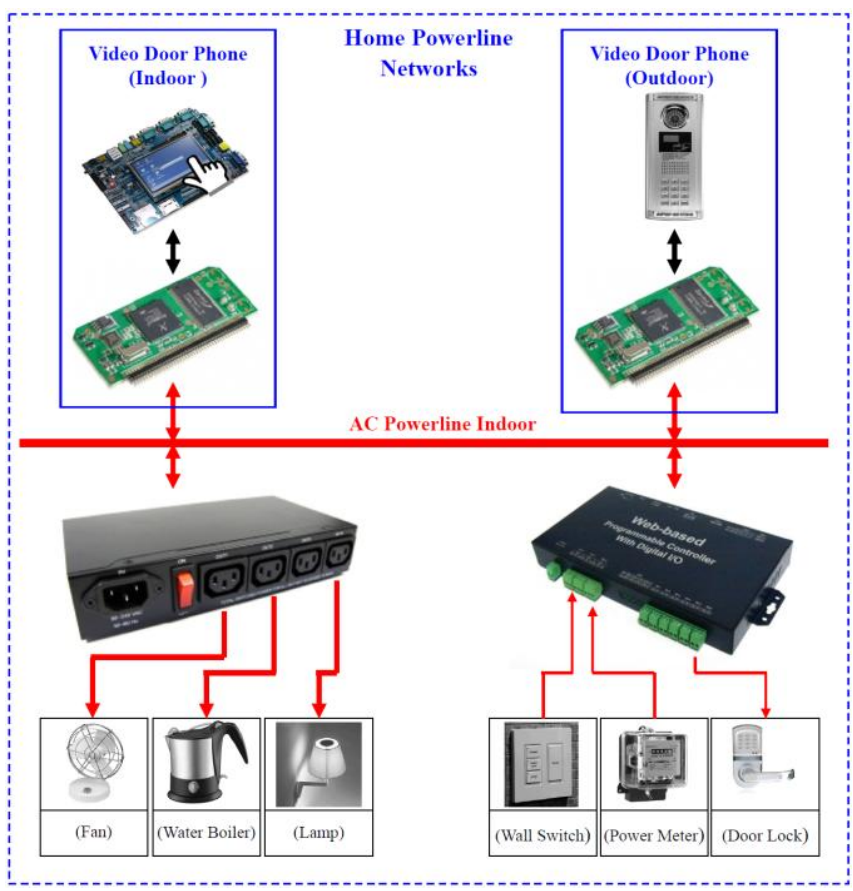

Fig. 9. Testing system of video door phone surveillance system

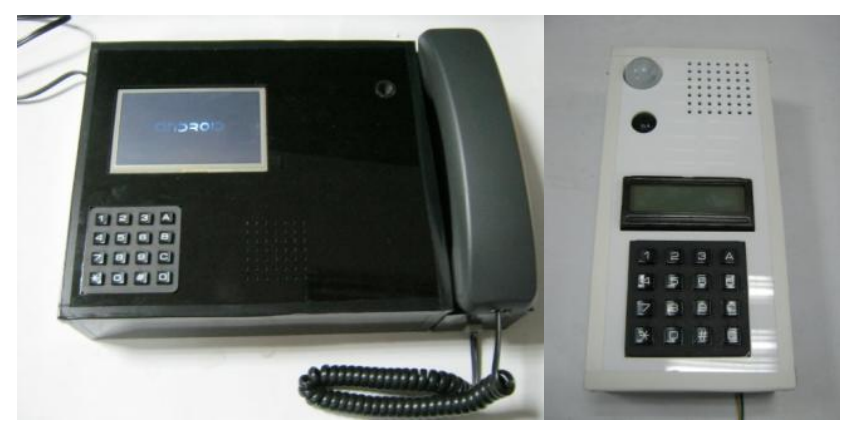

Fig. 10. Indoor and outdoor video phone

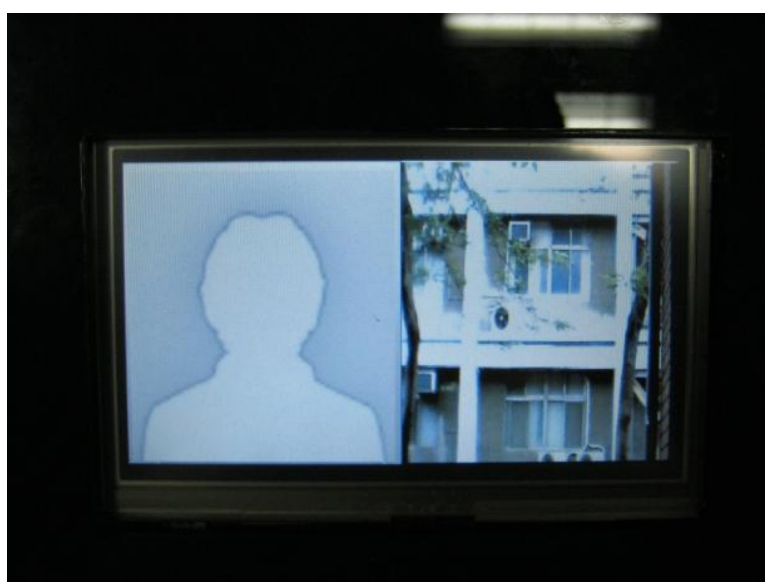

Fig. 11. Initial screen of indoor video phone

\section{CONCLUSION AND FUTURE WORKS}

Smart home is undoubtedly an important domain in the IT industry following the PC, PDA, and digital multimedia. Its technologies and applications are extremely vast, various home appliances can be controlled remotely through the home network, as well as sensor monitoring, surveillance and other applications.

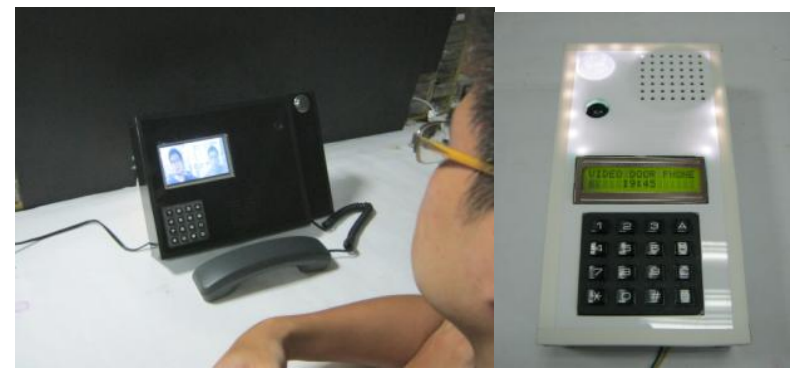

Fig. 12. Communication between indoor and outdoor

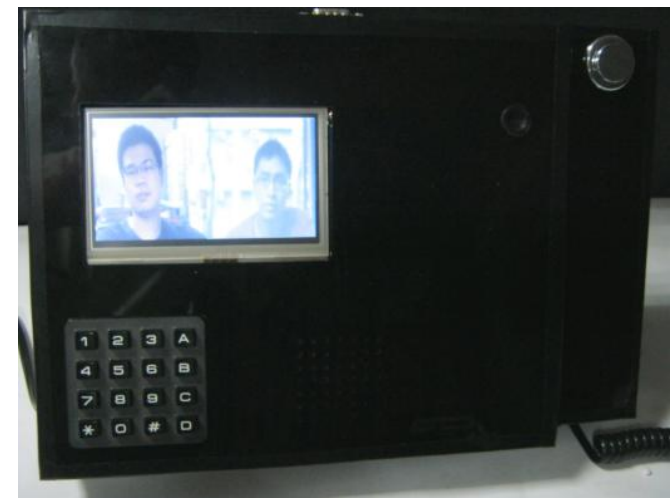

Fig. 13. Video screen on indoor phone while communicate to outdoor phone

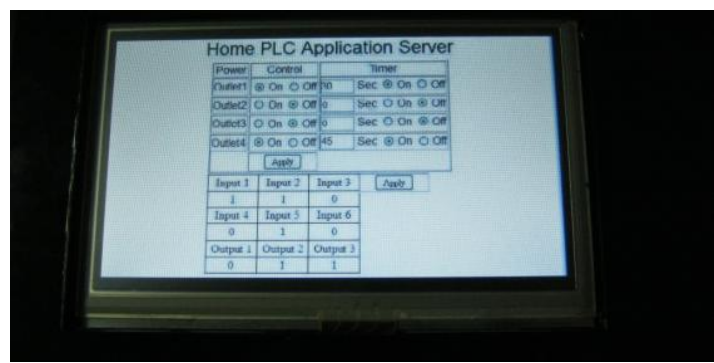

Fig. 14. User control interface on indoor video phone

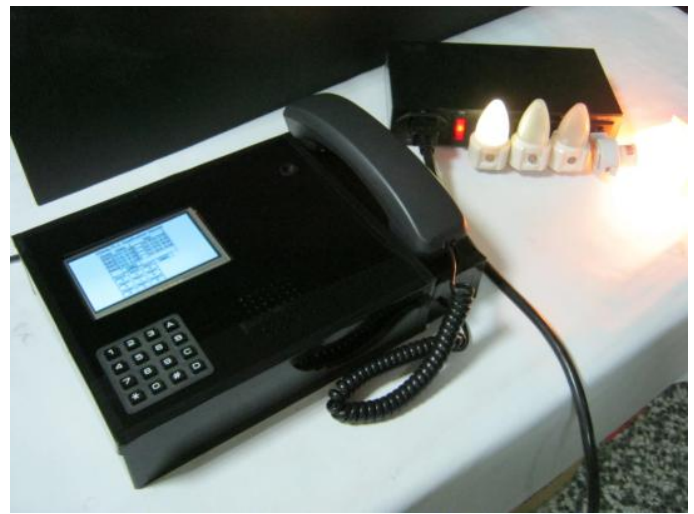

Fig. 15. Home appliances control via indoor video phone

In this home network, the power line is used as communication media following standard specifications of HomePlug AV and HomePlug C \& C V1.0. The economical video door phone system is then integrated into this network.

The implementation of this work is divided into four parts: construction of a video door phone system, construction of an indoor video phone server, porting embedded operating 
system, and system testing. The indoor and outdoor video phones are built with 32Bit ARM platform, and operated under the Android operating system. The outdoor video phone is coupled to the indoor phone to identify visitors, control door lighting and door locks. The indoor phone has a touch screen, which can facilitate the user to access smart home appliances connected on the power line, and record the image of entrance person with date/time information.

For the future works the outdoor video phone can be equipped with a touch screen to help hearing-impaired visitors to exchange information in sign language or handwritten text. The signal transfer over powerline communication across the phase, meter and transformer is still a problem, so for using this video door phone in community or between individual buildings, a bridge to link them should be developed.

\section{REFERENCES}

[1] City Buildings Will Sense and Respond Like Living Organisms, IBM, Dec. 2009

[2] T. B. Zahariadis, "Home Networking Technologies and Standards," Artech. House, 2003.

[3] W. Kastner, G. Neugschwandtner, S. Soucek, and H. M. Newmann, "Communication Systems for Building Automation and Control," in Proceedings of the IEEE, vol. 93, Issue 6, pp.1178 - 1203, Jun. 2005.

[4] J. Tu, W. W. Lin. J. C. Wang, and Y. T. Lin, "The Scenario Implementation of Home Networking," The 9th International Conference on Advanced Communication Technology, vol. 3, pp.1861 - 1863, Feb. 2007.

[5] V. Chunduru and N. Subramanian, "Effects of Power Lines on Performance of Home Control System," International Conference on Power Electronics, Drives and Energy Systems, PEDES '06, Dec. 2006.

[6] INSTEON Compared. (Jan. 2006). SmartLabs Technology. [Online] Available: http://www.smarthome.com/INSTEON_comparison.html

[7] Home Network Technology \& Connectivity Use: Ethernet, 802.11, Coax, and Powerline. (Sep. 2009). Global Information, Inc. [Online]. Available:

http://www.researchandmarkets.com/reports/1083481/home_network _technology_and_connectivity_use

[8] Powerline Technologies in Home Networking. (Jun, 2010). Xilinx Corp. [Online]. Available: http://www.xilinx.com/esp/consumer/home_networking/pdf_files/ch_ 7_plc/complete.pdf

[9] INT6400/INT1400 HomePlug AV Chip Set. (Dec. 2009). Datasheet, Atheros Powerline Technology. [Online]. Available: http://www.tomsnetworking.de/uploads/media/INT6400_INT1400_T echBrief.pdf

[10] HomePlug AV White Paper. (2005). HomePlug Powerline Alliance, Inc., [Online]. Available: http://www.homeplug.org/tech/whitepapers/HPAV-White-Paper_050 818.pdf
[11] HomePlug 1.0 Specification. (2001). HomePlug Powerline Alliance, Inc., Jun. [Online]. Available: http://read.pudn.com/downloads114/ebook/479147/HOMEPLUG.pdf

[12] M. K. Lee, R. E. Newman, H. A. Latchman, S. Katar, and L. Yonge, "HomePlug 1.0 Powerline Communication LANs - Protocol Description and Performance Results," International Journal of Communication Systems, vol. 16, pp. 1-6, 2000.

[13] S. Bradbury, "HomePlug Command \& Control (C\&C) Overview White Paper," Yitran Communications Ltd. and the HomePlug Command and Control Marketing Work Group, Sep. 2008.

[14] S5PC100 USER'S MANUAL (REV1.2), Samsung Electronics, Inc., Jun. 2009.

[15] P. Brady. (2008). Anatomy \& Physiology of an Android. Google I/O Session Videos and Slides. [Online]. Available: http://sites.google.com/site/io/anatomy--physiology-of-an-android

[16] Android 2.2, Release 1. (May 2010). [Online]. Available: http://developer.android.com/sdk/android-2.2.html/

[17] OpenCORE Media Framework. Android Open Source Project. (Aug. 2010). [Online]. Available: http://android.git.kernel.org/platform/external/opencore.git

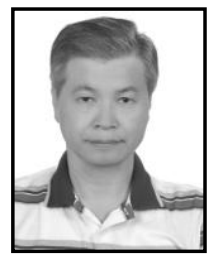

Chao-Huang Wei was born in Hsin-Chu, Taiwan 1951 Presently, he is an assistant professor with the Southern Taiwan University of Science and Technology, Tainan, Taiwan. He was graduated from National Centra University Taiwan with BS degree in Physics in 1974, and received Diplom Informatik degree from Institute of Informatik in 1984, and Doctor of Engineer degree from Institute of Microelectronics 1989, both from Technical University Berlin Germany.

From 1988 to 1994, he worked for several Taiwanese IT manufacturing companies as Project Manager, where he was involved in the developments of PC peripherals, entertainment equipments, digital camera, and Set Top Box. His current research areas include the development of smart home network, sensor network, and creation/innovation of daily necessities.

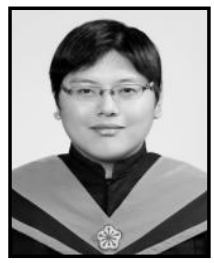

Ing. Wei has applied 45 patents and won 44 international invention awards in recent 6 years. The recent award is the National Prestige Enhancing Invention Order of Merit from World Inventor Award Festival 2012 in Seoul, Korea.

Shin-An Chen was born in Chia-Yi, Taiwan 1988. Presently, he is a graduate student a full time student in electrical engineering with the Southern Taiwan University of Science and Technology, Tainan, Taiwan.

From 2010 to 2013, he worked for several research projects in relation to home network systems as well as wireless sensor networking (WSN).

Mr. Chen has contributed his research efforts also for join in several national competitions in the area of intelligent household equipments and systems. 